\title{
Professionals' views on the use of smartphone technology to support children and adolescents with memory impairment due to acquired brain injury
}

\begin{abstract}
Purpose: To identify from a healthcare professionals' perspective whether smartphones are used by children and adolescents with acquired brain injury as memory aids; what factors predict smartphone use and what barriers prevent the use of smartphones as memory aids by children and adolescents. Method: A cross-sectional online survey was undertaken with 88 healthcare professionals working with children and adolescents with brain injury. Results: Children and adolescents with brain injury were reported to use smartphones as memory aids by $75 \%$ of professionals. However, only $42 \%$ of professionals helped their clients to use smartphones. The only factor that significantly predicted reported smartphone use was the professionals' positive attitudes towards assistive technology. Several barriers to using smartphones as memory aids were identified, including the poor accessibility of devices and cost of devices. Conclusion: Many children and adolescents with brain injury are already using smartphones as memory aids but this is often not facilitated by professionals. Improving the attitudes of professionals towards using smartphones as assistive technology could help to increase smartphone use in rehabilitation.
\end{abstract}




\section{Keywords}

Smartphone, brain injury, memory, rehabilitation, children, adolescents

\section{Introduction}

Prospective memory impairment, that is memory concerning planning for the future, is one of the most common and debilitating sequelae of acquired brain injury for children and adolescents [1-4]. Memory impairment can interfere with learning and access to education, everyday adaptive functioning and social participation [5-9]. Thus, interventions targeted at improving prospective memory are often employed in cognitive rehabilitation, via remedial or compensatory strategies [10]. The most common compensatory strategies used to support memory difficulties are paper and pencil external memory aids, including diaries $[1,11]$. However, these are limited in their utility as it is necessary to remember to check the reminder systems, which individuals with memory impairment find difficult $[1,4]$. In addition, young people report feeling self-conscious when using paper based memory aids. Alternatively, electronic external memory aids, such as personal data assistants (PDAs), are less conspicuous and have alarms which alert young people to check their reminder systems. In addition they often have multiple functions and applications that can support memory, such as calendars and voice recorders $[1,4]$. A number of studies have shown that electronic compensatory memory aids, such as pagers $[12,13]$, mobile phones $[14,15]$, PDAs $[1,10,16,17]$ and smartphones $[18,19,20]$ can be effective in supporting prospective memory in adults with brain injury, by helping them to remember and complete everyday tasks. A recent systematic review also concluded that external assistive devices reduce forgetfulness in adults after traumatic brain injury (TBI) or stroke [21]. However, the 
effectiveness of electronic devices at reducing forgetfulness in children and adolescents is unknown [5].

Recently, researchers have been particularly interested in the use of smartphones as assistive devices $[1,18,19,20]$. Smartphones are defined as mobile phones that have similar functions as a computer, including allowing users to run software applications [22]. Smartphones have become increasingly popular in memory rehabilitation because they have many functions and applications that can help people with memory impairment, for example calendars, alarms, to-do lists, texts and voice recorders [1,5]. Additionally, many children and adolescents already use smartphones, with $62 \%$ of $12-15$ year olds owning a smartphone in the UK [23]. Thus, for children and adolescents, smartphones could be viewed as desirable assistive devices as they are popular, inconspicuous and do not highlight their deficits to others $[5,18,22]$. However, to date only a few studies have provided evidence on the effectiveness of using smartphones as memory aids for children and adolescents with brain injury $[1,20]$. Gillette and DePompei $[20]$ found that students with TBI and intellectual difficulties, aging from six to 20 years, were more often on time and were more independent if they used a PDA or smartphone, compared to using a list or planner. However, this study was undertaken with a small sample of 35 students attending special educational settings, therefore the results may not be generalisable to children in mainstream education [20]. Additionally, this study only assessed the usefulness of the alarm function to prompt on time behaviour. Other functions of smartphones, such as to-do lists could also be useful in reducing forgetfulness [20]. Furthermore, Svoboda and colleagues [18] found using a single case experimental design that smartphone use increased 
confidence in memory demanding situations, reduced caregiver strain and increased quality of life in one 18 year old with memory impairment [18].

Despite the evidence to support the utility of assistive devices in memory rehabilitation, previous research has concluded that devices are under-utilised. One study found that only $36 \%$ of clinicians use portable electronic memory devices with TBI patients [24] and this percentage was likely to be an overestimate as participants were attendees of an assistive devices workshop and were interested in this subject. Indeed, a later study found the use of assistive devices to be lower with only $27.9 \%$ of professionals reporting use of assistive devices in treatment settings [25]. Additionally both studies reflect adult usage and refer to PDA and pager use which have now largely been replaced with smartphones as technology has progressed [24,25]. It is possible that smartphone usage may be higher due to their popularity. Thus, there is a need for more up to date research on the use of smartphones in rehabilitation, particularly for children and young people who increasingly own such devices.

The use of assistive technology in cognitive rehabilitation is affected by the usability and feasibility of using these devices in clinical practice. Clinician attributes may be important given that clinician training and confidence has been found to be linked to the rate of electronic memory device usage in a TBI sample [24]. Professionals' attitudes towards assistive technology and familiarity with smartphones could also affect device use by their clients [25]. Additionally, research has found that use of assistive devices is affected by factors unrelated to clinicians, such as the cost of devices and the unsuitability of devices for some patients $[4,24,25,26]$. However, given that research largely relates to adult populations it is unknown if there are additional barriers to using smartphones for young people, such as their parents' views and engagement with technology 
$[4,24,25,26]$. Further research is required to identify barriers specific to childhood populations to ascertain the feasibility of incorporating smartphones in rehabilitation programmes.

The objective of this research was to survey a wide range of professionals working with children and young people with brain injury in order to establish: 1) What is the reported usage of smartphones as memory aids for children and adolescents with brain injury?

2) What factors, such as professionals' attitudes towards assistive technology, experience, gender, age and familiarity with smartphones, predict the use of smartphones as memory aids by children and adolescents with brain injury? 3) What are the barriers to using smartphones as memory aids for children and adolescents with brain injury?

\section{Method}

\section{Study design}

This study used an online survey to elicit healthcare professionals' views on the use of smartphones as compensatory aids for children and adolescents with brain injury.

\section{Participants}

The target population consisted of professionals involved in the rehabilitation of children and adolescents with brain injury. One hundred and twenty participants consented to take part in the online survey and $88(66.7 \%)$ of these participants completed the survey. The demographic characteristics for the 88 participants are reported in table 1 . The respondents included a range of professionals working with children and adolescents with brain injury as detailed in table 1 . 
Many worked in hospitals but most reported working in other settings, such as further education colleges, private practice and charities. The majority of the respondents in the UK worked in the East Midlands $(n=31,44.3 \%)$ and those that worked outside the UK were mainly from the USA ( $n=14,15.9 \%)$, while two worked in Australia, one in Austria and one in Switzerland.

Insert table 1 about here

\section{Survey instrument}

The electronic survey was designed for this study based on previous research into clinicians' views on assistive technology $[24,25]$. The survey consisted of 20 questions and took approximately 10 minutes to complete. Participants were asked to provide demographic information, further to which they were asked about their familiarity with smartphone applications. Participants were asked whether they believed smartphones were being used as memory aids by the young people they worked with, what functions of the smartphones young people used to help them remember, what tasks young people used smartphones for and if they were aware of specific smartphone applications suitable to be used as memory aids. They were also asked whether they had helped the young people they worked with to use smartphones as memory aids. The survey included a six item scale used in previous research, the 'attitude towards assistive technology rating scale' [25]. This scale was validated in a previous study using factor analysis, one component was extracted with acceptable reliability (Cronbach's alpha $=0.70)[25]$. The questions in this scale were amended for the current study, so that smartphones were referred to in each question. A question was added to the scale later based on feedback but 
was not included in analysis to ensure the scale was valid. The internal consistency of the six item 'attitude towards assistive technology rating scale' used in this study was acceptable (Cronbach's alpha $=0.81$ ) [27]. The statements were answered on a 5-point scale (1: disagree, 2: slightly disagree, 3: no opinion/neutral, 4: slightly agree, 5: agree) with higher scores indicating more positive attitudes. The final section asked participants to judge the importance of several barriers to using smartphones. The barriers were rated on a 5 point scale (1: not significant at all, 2: somewhat insignificant 3: no opinion/neutral, 4: somewhat significant 5: very significant) with higher scores indicating the barrier was more significant.

A first draft of the survey was piloted by administering the online survey to 15 professionals, including Occupational Therapists, Health Psychologists, Assistant Psychologists and Clinical Neuropsychologists. Following feedback, some minor amendments were made.

\section{Procedure}

Ethical approval was received for this study from the University of Nottingham, School of Medicine Research Ethics Committee. The survey was distributed by email to administrators of three mailing lists: Child Brain Injury Trust mailing list and a UK and international professional email network for child neuropsychologists. Additionally, the survey was emailed to professional contacts. Prior to commencing the survey participants were presented with an information sheet and were informed that continuation with the survey would be an indication of consent. 


\section{Data analysis}

To assess reported smartphone use descriptive statistics such as frequencies and percentages were used. Chi Square tests and Mann-Whitney U-tests were used to determine if there was a relationship between smartphone use and other variables. Logistic regression analysis was used to assess which variables predict reported smartphone use. Smartphone use was coded so that $0=$ does not use smartphones and $1=$ uses smartphones. Additionally, to assess the barriers to using smartphones as memory aids descriptive analysis was undertaken. The data were analysed using SPSS for Windows, Version 21.

\section{Results}

\section{Smartphone use}

Three-quarters $(n=66)$ of professionals reported that they were aware that the children and adolescents they worked with used smartphones as memory aids. Sixty-seven \% ( $n=59)$ of professionals reported discussing the use of smartphones as memory aids with the young people they work with. However, only $42 \%(n=37)$ reported helping the children and adolescents they work with to use smartphones as memory aids. Professionals reported that young people used the calendar, alarm and reminder functions of the smartphone the most and other applications the least; other applications included maps and internet. Furthermore, professionals reported that the tasks young people used a smartphone for, were mostly keeping in touch with friends and they used a smartphone least when managing money. Only $26.1 \%(n=23)$ of professionals 
were aware of a specific smartphone application suitable as a compensatory memory aid for children and adolescents with memory difficulties. Most professionals ( $n=78,88.6 \%$ ) reported that they would be willing to use a smartphone application with the young people that they work with if it was adapted for young people with brain injury. The most common adaptations professionals suggested were: larger font size, simpler navigation, the condensing of existing functions into one application, more age appropriate, voice recording options, personalisation and relating applications to school. Only $26.1 \%(n=23)$ of professionals were aware of a specific smartphone application suitable as a compensatory memory aid for children and adolescents with memory difficulties.

\section{Predictors of smartphone use}

The data for both the attitudes variable and the familiarity variable was negatively skewed, had kurtosis and was also found to be significantly nonnormal by the Kolmogorov-Smirnov (K-S) test. Therefore the median and interquartile ranges were reported and non-parametric analysis was undertaken [27]. Descriptive analysis revealed that respondents overall had neutral to slightly positive attitudes towards assistive technology (median $=3.33$, IQR $=$ .80). Respondents also had a high level of familiarity with using smartphones $($ median $=5.00, \mathrm{IQR}=1.00)$, suggesting that respondents used smartphones often.

Mann-Whitney U-tests were carried out to test the magnitude of the relationships between professionals' awareness of the young people they worked with using smartphones as memory aids, professionals' attitudes towards assistive technology and professionals' familiarity with smartphones. As shown in 
table 2, the Mann-Whitney U-tests indicated that professionals' attitudes towards assistive technology were significantly more positive for those professionals who reported that the young people they worked with used smartphones as memory aids than those that did not. However, the professionals' familiarity with smartphones did not significantly differ between groups. Additionally, a MannWhitney U-test found that, professionals' attitudes towards assistive technology were significantly more positive for those professionals who reported that they helped the young people they worked with to use smartphones as memory aids $($ median $=3.83, \mathrm{IQR}=0.8)$ than those that $\operatorname{did}$ not $($ median $=3.17, \mathrm{IQR}=1)$, $U=614.00, z=-2.80, p<0.01, r=-0.30$. Chi square analysis was undertaken to determine if there were any associations between the demographics of the professionals and professionals' awareness of the young people they worked with using smartphones as memory aids. There were no significant associations between smartphone use and age, gender, experience, working in the UK, professional setting and profession.

Insert table 2 about here

Logistic regression analysis was used to determine which factors predicted smartphone use by children and adolescents with brain injury, as reported by professionals, and is summarised in table 3. In the first block, demographic variables which could have been potential confounders, as identified in previous literature $[20,22]$, were entered in the logistic regression as categorical variables. These variables included: respondents' age, gender and years of professional experience. In the second block professionals' familiarity with using smartphones and professionals' attitudes towards assistive technology were 
entered into the model. The Hosmer and Lemeshow test was non-significant, indicating that the model including all variables was a good fit of the data, $x^{2}(7)$ $=4.64, p=0.70$. The Nagelkerke's $\mathrm{R}^{2}$ of 0.35 showed that all the variables in the model predicted $35 \%$ of the variability of smartphone use and was therefore not a good model. Additionally, professionals' attitudes towards assistive technology were the only significant predictor of smartphone use, with the other variables included in the model, $b=2.25$, Wald $x^{2}=11.53, p<0.001$. The odds ratio showed that professionals who had positive attitudes towards using smartphones as memory aids were $9.46\left(95 \% \mathrm{CI}^{\prime} \mathrm{s}=2.59\right.$ to 34.64$)$ times more likely to have reported that their clients used smartphones as memory aids than those that did not, even after accounting for confounding variables. The sensitivity of the results was $92.3 \%$, the specificity was $36.4 \%$ and $78.2 \%$ were correctly classified.

\section{Insert table 3 about here}

\section{Barriers}

The medians (M) and interquartile ranges (IQR) of the barriers were calculated and revealed that professionals reported poor accessibility due to a small screen size or keyboard $(M=4 ; \mathrm{IQR}=0.75)$; cost of the device $(M=4 ; \mathrm{IQR}=0)$; vulnerability to loss, theft or bullying $(M=4$; IQR $=2)$; parents' lack of engagement with technology $(M=4 ; I Q R=1)$ and their clients' inability to use smartphones independently $(M=4 ; \mathrm{IQR}=1)$, as the most significant barriers to using smartphones in memory rehabilitation. Professionals reported that the least significant barrier to using smartphones as compensatory memory aids was that it would be unfair on siblings who did not have a smartphone $(M=2 ; \mathrm{IQR}=$ 
2). Mann-Whitney U-tests were carried out to investigate whether there were any associations between professionals reported use of smartphones by their clients and professionals' beliefs in the significance of each barrier. However, the results showed that none of the barriers were related to smartphone use ( $p=$ 0.13 to 0.98$)$.

\section{Discussion}

This study found that three quarters of professionals reported that the children and adolescents with brain injury that they worked with used smartphones as memory aids but only $42 \%$ of professionals helped the young people they worked with to use smartphones. This percentage is similar to previous studies, which found that that only $27.9 \%$ [25]-36\% [24] of the professionals surveyed used portable electronic memory devices with their patients. Therefore, while most children and adolescents were found to be using smartphones as compensatory memory aids, many do this without support from professionals. This suggests that there is further scope to incorporate this potentially beneficial technology into rehabilitation to ensure that more people with memory impairment can benefit from this technology. A study by DePompei and colleagues [1] offers insight into how smartphones could be implemented into rehabilitation in their suggested intervention plan for students with memory and organisational problems. This intervention plan would undoubtedly need the assistance of family or support workers to help with the set up and use of the applications. 
Additionally, the current study found that according to healthcare professionals, children and adolescents with brain injury primarily used the calendar, alarm and reminder functions of smartphones and mainly used smartphones to keep in touch with friends. This is similar to the findings of other studies that asked students with brain injury what functions and tasks they used their PDAs or smartphones for $[1,20]$.Thus, there may be potential for supporting young people in the use of further applications that could help them with a variety of tasks or for the development of more specific applications to support young people with brain injury.

The only significant predictor of children and adolescents reported use of smartphones as memory aids was professionals' attitudes towards assistive technology. Professionals who had positive attitudes towards using smartphones as memory aids were more than nine times more likely to have reported that their clients used smartphones as memory aids than those that did not, even after accounting for confounding variables such as age, gender and years of experience. However, only $35 \%$ of the variance in smartphone use was explained by this regression model, which indicates that there are other important factors that need to be investigated, such as parents' attitudes and familiarity with smartphones [25]. These results concur with previous research, which found that professionals' confidence in teaching participants how to use devices was a significant predictor of smartphone use in rehabilitation [24]. Indeed, the attitudes towards assistive technology scale used in the current study included an item in which respondents had to rate how confident they were helping a patient to work with a smartphone, which is similar to the confidence measure used in previous research [24]. However, the current study suggests that there are other significant factors, beyond clinicians' confidence in 
training, such as their general attitudes towards assistive devices that could impact on smartphone use. Additionally, previous research did not find that personal use of devices by professionals predicted smartphone use with patients [24]. Similarly, the current study found professionals' familiarity with smartphones did not predict smartphone use. Furthermore, in the current study, a significant association was found between positive attitudes towards assistive technology and helping young people with brain injury to use smartphones as memory aids. Based on the results of this study and previous research, it can be suggested that improving the confidence of professionals to teach those with brain injury how to use devices and improving professionals' attitudes towards smartphones as assistive technology, could help to increase the use of smartphones in memory rehabilitation and potentially improve the amount of help children receive from professionals to use these devices [24]. Professionals' attitudes and confidence in using technology in rehabilitation could be improved via provision of training aimed at increasing their knowledge of how technology can be used to improve care. This could include hands on training on how to incorporate technology within intervention plans, such as that suggested by DePompei and colleagues [1]. Additionally more research on the effectiveness of smartphone applications in improving memory, increasing autonomy and reducing caregiver strain would likely improve professionals' attitudes towards assistive technology.

This study also found that professionals believed that the most significant barriers to using smartphones as memory aids were: the poor accessibility of smartphones; cost of smartphones; vulnerability to loss; theft or bullying; parents' lack of engagement with technology and the inability of some young people to use smartphones. Many of these barriers have also been identified in 
previous research on PDAs as major barriers to device use $[25,28]$. Poor accessibility could be addressed by using tablet computers as an alternative to smartphones, as these devices have much larger screens and keyboards. However, tablets may be less portable and more conspicuous than smartphones due to their larger size, which may deter young people from using them. The lack of parents' engagement could also be addressed by demonstrating to parents the benefits of using assistive devices. Previous studies have also identified other barriers not assessed in this research, such as the professionals' inability to facilitate device use in a natural setting and client motivation and insight [26]. Additionally, the appropriateness of current smartphone applications for children and adolescents with brain injury could also be seen as a barrier to their use. Indeed, this study found that most professionals $(88.6 \%)$ would be willing to use a smartphone application with the young people that they work with if it was adapted for young people with brain injury and very few (26.1\%) were aware of a current application that was appropriate. Thus, the development of an application appropriate for this population could enhance the utility of smartphones as compensatory aids. However, despite these barriers, using smartphones as memory aids in rehabilitation could have benefits over standard procedures, such as PDAs or paper based reminder systems. For example, smartphones may facilitate social inclusion if they could assist young people with memory impairment in being able to navigate their world more confidently without highlighting their deficits to others. Using assistive technology could also assist with the development of autonomy during adolescence, thereby increasing the self-esteem of the young person and subsequently reducing caregiver strain. 


\section{Limitations and Strengths}

A limitation of this study was that it was necessary to utilise a novel survey so the validity and reliability of the questionnaire has not been evaluated. The cross-sectional survey design also did not allow for a causal relationship between positive attitudes towards using assistive technology and reported smartphone use to be established. Additionally, smartphone use by young people was only measured by professionals' reports of smartphone use and was not reported by the young people themselves. However, it was important to understand professionals' views on smartphone use in rehabilitation, as smartphones are unlikely to be used in rehabilitation without the support of professionals [24].

A risk of sampling bias should be acknowledged in that those who completed the survey may have had a greater interest in using smartphones in rehabilitation and as a consequence be more familiar with smartphones and have more positive attitudes towards using smartphones as assistive devices. Additionally, the sample may be biased as it consisted of mostly females, aged 35-54, with 5-10 years of experience, which may not be representative characteristics of the wider population of professionals. Also, the sample size may have been too small to identify all the predictor variables and could have contributed to the poor fit of the model to the data. However, the sample size was similar to previous surveys $[24,25,26]$.

Despite these limitations the sample in this study was geographically varied with participation healthcare professionals from across the UK, as well as some from outside of the UK. This suggests that the results of this study may be less biased by geographical variations in healthcare provision. Furthermore, this study gathered data from a wide range of professionals involved in the rehabilitation of children and adolescents with brain injury, including medical 
professionals, psychologists and teachers. Thus, the views of professionals gathered in this study are likely to reflect the views of the many different professions involved in rehabilitation. Importantly this research has also contributed to a growing area of research into the use of assistive devices in memory rehabilitation for children and adolescents with brain injury and has offered insight into how smartphone use could be increased in cognitive rehabilitation.

\section{Future research}

Future research with young people who have brain injury is required to further understand the support young people may require in using smartphones as compensatory aids. It is also necessary to understand young people's views on using smartphones as a memory aid and what applications they would find most useful. A feasibility trial would be needed to assess the efficacy of using smartphones in rehabilitation and identify problems. It could also be beneficial to determine whether other factors, such as parents' attitudes towards assistive technology predict smartphone use. Additionally, future research could investigate whether providing workshops aimed at improving professionals' attitudes towards smartphones as assistive devices has an impact on smartphone use and the amount of help with smartphones professionals' provide to their clients. Research could also address some of the barriers to using smartphones, such as establishing whether tablets are more accessible than smartphones; or whether improving parents' engagement and knowledge of smartphones would help to improve use. The development of a new smartphone application that incorporates the suggestions that professionals in this study made and piloting this application in rehabilitation services for children and 
adolescents with brain injury could also help to produce a stronger evidence base for the use of smartphones in cognitive rehabilitation.

\section{Conclusion}

Prospective memory impairment is one of the most common sequelae of brain injury and can have a debilitating effect on the lives of young people with brain injury [1-9]. Research has suggested that using smartphones as compensatory aids can help to improve functioning in children and adolescents $[1,18,20]$. The findings of this online survey were that most children and adolescents with brain injury were reported to be using smartphones as compensatory aids but few were receiving help from professionals to use smartphones as memory aids. Furthermore, it was found that the more positive a professionals' attitude towards using smartphones as assistive devices, the more likely it was that they would report the young people that they worked with used smartphones as memory aids. Additionally, a more positive attitude towards smartphones as an assistive device was associated with professionals helping young people to use smartphones as memory aids. Thus, improving the attitudes of professionals towards assistive technology could increase smartphone use and the support that young people with brain injury receive to use smartphones as memory aids. However, professionals also identified that there were many barriers to using smartphones as memory aids, such as the poor accessibility and cost of devices. Future research should focus on young people's views regarding using smartphones as memory aids, including the design of bespoke applications and subsequently the measurement of the efficacy of such technology within the population. 


\section{Declaration of interests}

The authors report no declarations of interest.

\section{References}

1. DePompei R, Gillette Y, Goetz E, et al. Practical applications for use of PDAs and smartphones with children and adolescents who have traumatic brain injury. NeuroRehabilitation 2008; 23: 487-499.

2. Lezak MD, Howieson DB, Bigler ED, et al. Neuropsychological Assessment. New York, Oxford: Oxford University Press, 2012.

3. Middleton, J. A. Brain injury in children and adolescents. Advances in Psychiatric Treatment 2001; 7: 257-265.

4. Ptak $R$, Van der Linden $M$ and Schnider $A$. Cognitive rehabilitation of episodic memory disorders: from theory to practice. Frontiers in human neuroscience $2010 ; 4: 1-11$.

5. Linden M, Hawley C, Blackwood B, et al. Technological aids for the rehabilitation of memory and executive functioning in children and adolescents with acquired brain injury. Cochrane Database Syst Rev 2014, Issue 3.

6. Ewing-Cobbs L, Barnes M, Fletcher JM, et al. Modeling of longitudinal academic achievement score after pediatric traumatic brain injury. Developmental Neuropsychology 2004; 25:107-33.

7. Hawley $C A$, Ward $A B$, Magnay $A$, et al. Return to school after brain injury. Archives of Disease in Childhood 2004; 89:136-142.

8. Anderson V, Beauchamp M, Yeates KO, et al. Social competence at 6 months following childhood traumatic brain injury. Journal of the International Neuropsychological Society 2013;19(5):539-50. 
9. Bedell GM and Dumas HM. Social participation of children and youth with acquired brain injuries discharged from inpatient rehabilitation: a followup study. Brain Injury 2004;18:65-82.

10.Lannin N, Carr B, Allaous J, et al. A randomized controlled trial of the effectiveness of handheld computers for improving everyday memory functioning in patients with memory impairments after acquired brain injury. Clinical rehabilitation 2014; 1-12.

11.Sohlberg, M Kennedy M, Avery J, et al. Evidence-based practice for the use of external aids as a memory compensation technique. Journal of Medical Speech-Language Pathology 2007; 15:xv-li.

12. Wilson BA, Emslie HC, Quirk K, et al. Reducing everyday memory and planning problems by means of a paging system: a randomised control crossover study. Journal of neurology, neurosurgery, and psychiatry $2001 ; 70: 477-82$

13. Wilson BA, Emslie $H$, Quirk $K$, et al. A randomized control trial to evaluate a paging system for people with traumatic brain injury. Brain Injury 2005; 19: $891-894$.

14.Stapleton S, Adams M and Atterton L. A mobile phone as a memory aid for individuals with traumatic brain injury: A preliminary investigation. Brain injury 2007; 21:401-411.

15. Wade TK and Troy JC. Mobile phones as a new memory aid: a preliminary investigation using case studies. Brain injury 2001, 15:305-320.

16. Dowds MM, Lee PH, Sheer JB, et al. Electronic reminding technology following traumatic brain injury: effects on timely task completion. The Journal of Head Trauma Rehabilitation 2011; 26: 339-347. 
17. Gentry T, Wallace J, Kvarfordt C, et al. Personal digital assistants as cognitive aids for individuals with severe traumatic brain injury: A community-based trial. Brain Injury 2008; 22: 19-24.

18.Svoboda E, Richards B, Polsinelli A, et al. A theory-driven training programme in the use of emerging commercial technology: Application to an adolescent with severe memory impairment. Neuropsychological rehabilitation $2010 ; 20: 562-86$.

19.Svoboda E, Richards B, Leach L, et al. PDA and smartphone use by individuals with moderate-to-severe memory impairment: application of a theory-driven training programme. Neuropsychological rehabilitation $2012 ; 22: 408-27$.

20. Gillette $Y$ and Depompei R. Do PDAs enhance the organization and memory skills of students with cognitive disabilities? Psychology in the Schools 2008; 45: 665-677.

21. Cicerone KD, Langenbahn DM, Braden C, et al. Evidence-based cognitive rehabilitation: updated review of the literature from 2003 through 2008. Arch Phys Med Rehabil 2011; 92:519-530.

22. Luxton DD, McCann RA, Bush NE, et al. mHealth for mental health: Integrating smartphone technology in behavioural healthcare. Professional Psychology: Research and Practice 2011; 42: 505-512.

23. The Office of Communications. Younger children turn from phones to tablets. http://media.ofcom.org.uk/news/2013/younger-children-turnfrom-phones-to-tablets/ (2013, accessed 28 August 2014).

24. O'neil-pirozzi TM, Kendrick H, Goldstein R, et al. Clinician influences on use of portable electronic memory devices in traumatic brain injury rehabilitation. Brain injury 2004; 18: 179-189. 
25. de Joode EA, van Boxtel MP, Verhey FR, et al. Use of assistive technology in cognitive rehabilitation: Exploratory studies of the opinions and expectations of healthcare professionals and potential users. Brain Injury 2012; 26: 1257-1266.

26. Speaks KL. A Survey of Assistive Technology in Cognitive Rehabilitation. MA Thesis, University of Minnesota, USA, 2013.

27. Field A. Discovering Statistics Using SPSS, $3^{\text {rd }}$ ed. London: Sage publications, 2009

28. Hart T, Buchhofer R, and Vaccaro M. Portable electronic devices as memory and organizational aids after traumatic brain injury: a consumer survey study. The journal of head trauma rehabilitation 2004; 19: 351365.

29. Evans JR and Mathur A. The value of online surveys. Internet Research 2005; 15: 195-219. 